\title{
APORTE DE FUENTES DE AGUAS SUPER- FICIALES EN LA PARTE ESTE DEL LAGO ENRIQUILLO Y EL IMPACTO DE ESAS AGUAS EN EL CRECIMIENTO DEL LAGO
}

Contribution of surface water sources in the eastern part of Lake Enriquillo and the impact of these waters to the growth of the lake

\section{Virgilio Antonio Miniño Mejía* Yolanda León Cándido Quintana Aida Rosario}

Resumen: El lago Enriquillo es el más grande de las Antillas con una extensión superficial de aproximadamente $265 \mathrm{~km}^{2}$ (actualmente se aproxima a los $400 \mathrm{~km}^{2}$ ). La salinidad del lago había sido hasta hace poco tiempo mucho mayor que la del mar, pero el aumento de su nivel de aguas ha bajado la salinidad del mismo considerablemente hasta unas 20 partes por mil. Está situado a aproximadamente 44 metros bajo el nivel del mar. Su crecimiento desproporcionado ha causado alarma en la comunidad científica internacional, pues es algo sin precedentes hasta donde se conoce. Durante su historia el lago Enriquillo ha experimentado oscilaciones en el nivel de sus aguas, pero durante esta última crecida, a

Profesor investigador del Instituto Tecnológico de Santo Domingo (INTEC) Correo electrónico: Virgilio.minino@intec.edu.do 
partir del año 2004, este crecimiento ha sido muy rápido y continuo, lo cual ha provocado cierta incertidumbre entre los científicos que han trabajado la geología, la hidrología y la hidrogeología del área, pues no se ha podido determinar la causa exacta de este fenómeno.

Palabras clave: Lago Enriquillo, salinidad, hidrología, hidrogeología, agua superficial.

Abstract: Lake Enriquillo is the largest of the Antilles with a surface area of approximately $265 \mathrm{~km}^{2}$ (now approaching $400 \mathrm{~km}^{2}$ ). Until recent time the salinity of the lake had been much greater than sea's salinity, but the increased of its water level has dropped considerably its salinity to $20 \mathrm{ppt}$. It is located about 44 meters below sea level. Its disproportionate growth has alarmed the international scientific community, and it is unprecedented as far as is known. During its history the Enriquillo lake has experienced fluctuations in the level of the water, but during the last flood, from 2004, this growth has been rapid and continuous, which has caused some uncertainty among the scientists who worked the geology, hydrology and hydrogeology of the area because it has not been possible to determine the exact cause of this phenomenon.

Keywords: Lake Enriquillo, salinity, hydrology, hydrogeology, surface water. 
Aporte de fuentes de aguas superficiales en la parte Este del Lago Enriquillo y el impacto de esas aguas en el crecimiento del lago

\section{Introducción}

La República Dominicana se encuentra ubicada entre los $17^{\circ} 36^{\prime}$ $19^{\circ} 58^{\prime}$ latitud Norte y los $68^{\circ} 19^{\prime}-72^{\circ} 01^{\prime}$ longitud Oeste, y ocupa unas dos terceras partes de la isla de La Española (Hispaniola), Antillas Mayores, con una extensión de unos 48,442 km². Figura N. ${ }^{\circ}$ 1. El lago Enriquillo se encuentra en el suroeste del país entre las sierras de Neyba y Bahoruco, y es el más grande de las Antillas con una extensión superficial de aproximadamente 265 $\mathrm{km}^{2}$ (Mann et al., 1991; Koehler et al., 2013), y que actualmente se aproxima a los $400 \mathrm{~km}^{2}$ debido al continuo crecimiento del nivel de sus aguas.

\section{Figura N. ${ }^{\circ} 1$}

\section{Mapa de la República Dominicana y el lago Enriquillo}

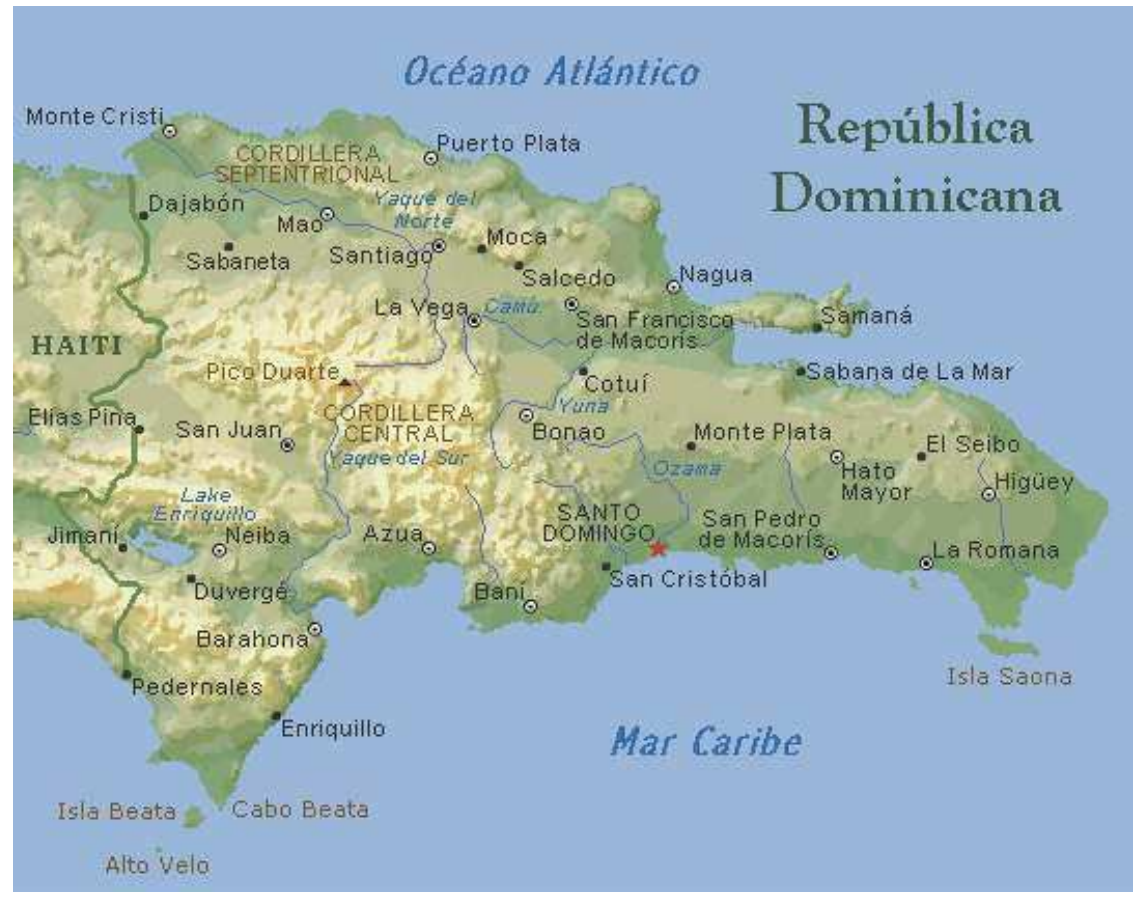

Fuente: Google. 
Dentro del lago se encuentran tres islas. La isla Cabrito, que es la mayor tiene un área de unos $24 \mathrm{~km}^{2}$. También están las islas Barbarita y la Islita. Véase la figura N. ${ }^{\circ}$ 2. La salinidad del lago había sido hasta hace poco tiempo mucho mayor que la del mar (Buck et al., 2005), pero el aumento de su nivel de aguas ha bajado la salinidad del mismo considerablemente hasta unas 25 partes por mil (INTEC 2013). Datos más recientes y no publicados dan una salinidad de 30 partes por mil, lo cual indica que el lago ha estado recibiendo menos aguas dulces y que el nivel de sus aguas ha bajado, como también lo muestran las más recientes mediciones, aun sin publicar. El lago forma parte del Parque Nacional Lago Enriquillo e Isla Cabritos así como de la Reserva de la Biosfera de JaraguaBahoruco-Enriquillo.

\section{Figura N. ${ }^{\circ} 2$}

\section{Mapa local del Lago Enriquillo}

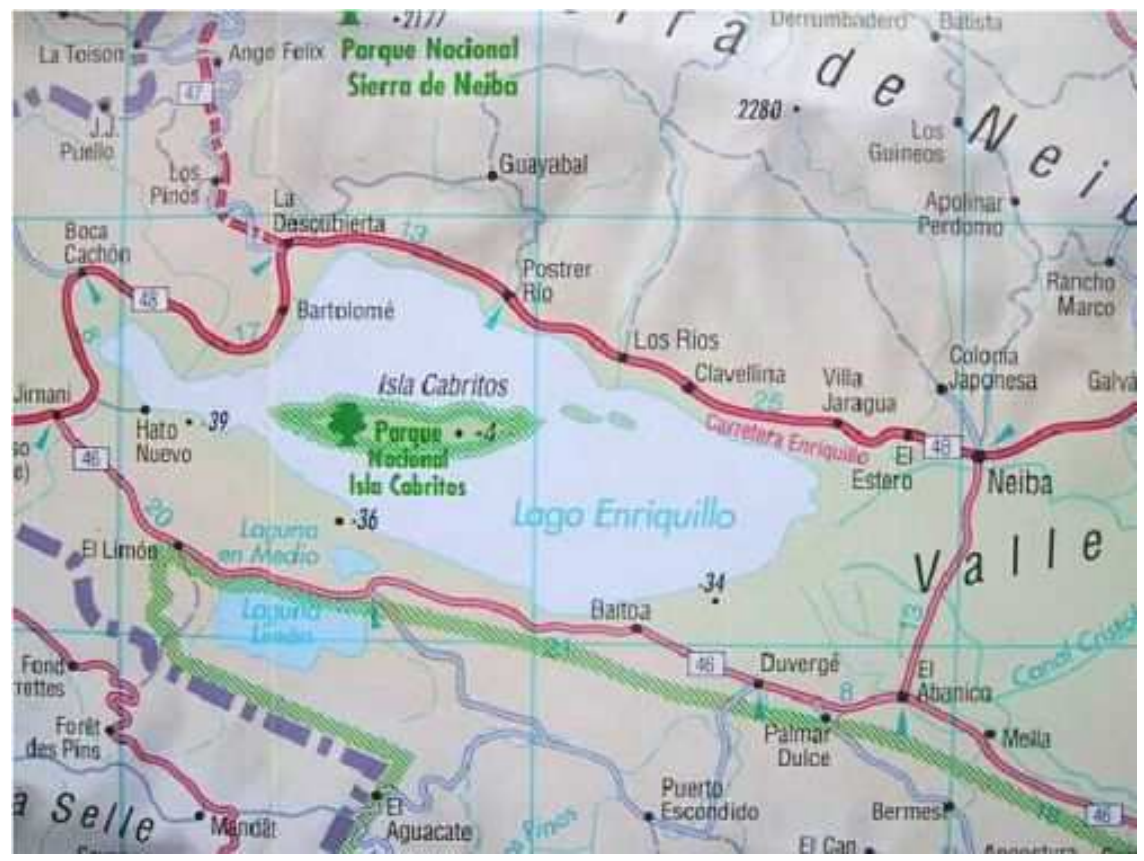

Fuente: Google. 
Múltiples estudios se han estado realizando alrededor del lago Enriquillo, aportando valiosas informaciones científicas sobre su comportamiento. Entre estos estudios podemos citar los siguientes: Antonio Cocco Quezada, "El ciclo hidrológico del lago Enriquillo y la crecida extrema del 2009"; Huáscar Martínez, "Consideraciones acerca del riesgo de inundación del poblado de Jimaní. Riesgo de Inundación debido al rebose del lago Saumatre o Azuei hacia el Lago Enriquillo", 2010; Medrano y Payano, “Análisis de la situación actual del lago Enriquillo de la República Dominicana. Instituto IMDEA Agua", 2011; Luna Romero, "Water Level Fluctuations of Lake Enriquillo and Lake Saumatre in Response to Environmental Changes", 2011, entre otros.

\section{Geología e hidrogeología del Lago Enriquillo}

Desde el punto de vista geológico-tectónico el lago Enriquillo, al igual que la laguna de Cabral y el lago Sumatra en Haití, es interesante pues es el resultado de un canal marino que en tiempos geológicos comunicaba la bahía de Neyba con Puerto Príncipe. (Mann, 1991).

El lago Enriquillo recibe una gran influencia de manantiales, ríos y cañadas que proveen parte de su agua a través del subsuelo. Entre estos están: Boca de Cachón, Las Marías, La Zurza, Las Barías, río Guayabal, río Las Damas, río Bermesí, y río Barreras, contribuyendo al lago aproximadamente 1,100 millones $\mathrm{m}^{3} /$ año (Mann, 1991, De León, 2012, Gil et al., 2012). Esta abundancia de agua subterránea podría explicarse por la peculiar geología de las sierras que rodean al lago. Estudios de Etypsa (2004) indican que la Sierra de Bahoruco "está formada por un sistema de calizas cenozoicas de estructura compleja, falladas y con formaciones cársticas de gran magnitud, cuya cara norte (la más escarpada de la sierra) está separada de la Hoya del Lago Enriquillo por una escalera de fallas". Véase la figura N. ${ }^{\circ}$. Esta afirmación es de gran importancia para las investigaciones del área, pues hace evidente la influencia que tiene la geología en los sistemas de desagüe hacia el lago. 
Figura $\mathrm{N}^{\circ} 3$

Rocas calizas

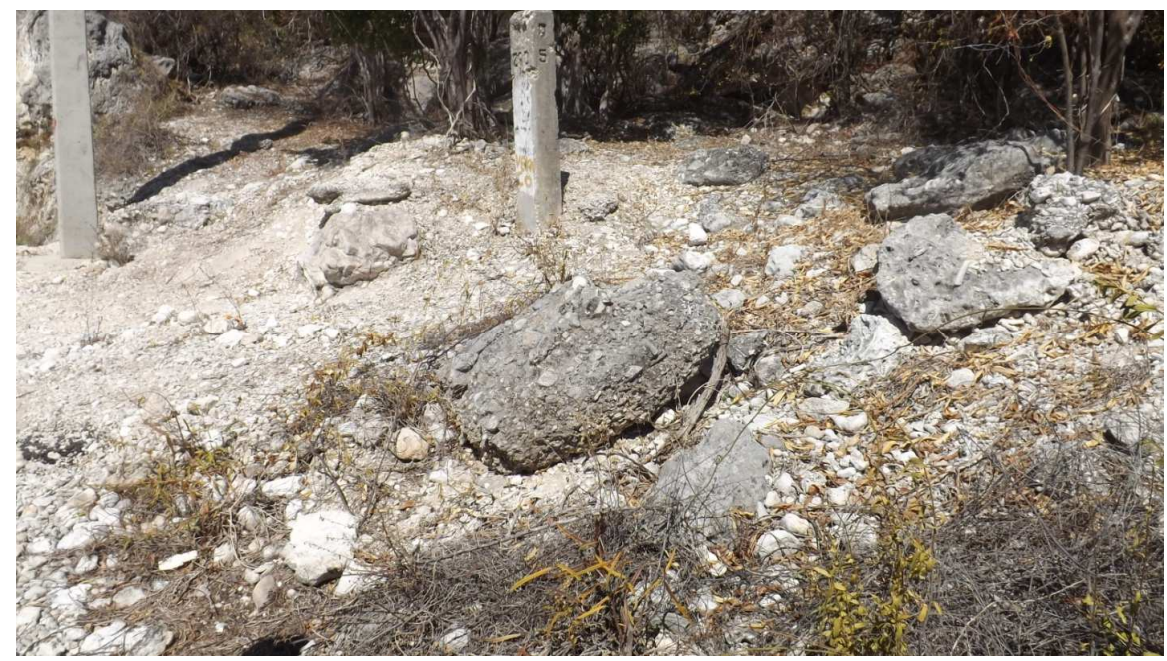

\section{La crecida del lago: Hipótesis}

La crecida del lago Enriquillo a partir de 2004 ha generado un debate entre investigadores y se han propuesto múltiples teorías sobre este fenómeno. Payano \& Medrano (2011) indican que algunos de los factores atribuidos al aumento en el nivel del agua de los lagos de la región son la deforestación, consecuencias del cambio climático, mala gestión y mal estado de las infraestructuras hidráulicas que drenan en la cuenca Enriquillo, eventos climáticos, y la geología y movimientos tectónicos. Ciertamente que las investigaciones han dado diferentes resultados y ha sido necesario seguir obteniendo y tabulando datos para poder llegar a una hipótesis consensuada.

Algunos autores han señalado que el lago Enriquillo históricamente ha tenido períodos de crecimiento seguidos de períodos de descenso (Noble, 1929, Ducoudray, 1981). Varios autores han planteado que las fluctuaciones en el nivel del lago Enriquillo están directamente relacionadas con eventos meteorológicos (Payano 
\& Medrano 2011; Herrera \& Orrego 2011, Cocco-Quezada 2009, González 2012 \& Schubert, 2012), entre otros. Todos ellos coinciden en el hecho de que los fenómenos meteorológicos extremos que afectaron la región suroeste de la República Dominicana a partir del 2004 han sido responsables, al menos en parte, de la crecida del lago Enriquillo.

Jiménez \& Galizia (2012), y Gil et al. (2012) consideran que "la tendencia histórica del nivel del lago ha sido por mucho tiempo considerada a disminuir debido a que las aguas que lo alimentan estaban siendo usadas en la agricultura y consumo humano en los pueblos asentados en la región, mientras que la evaporación es constante". Gil et al. (2012) también añaden que este patrón ha sido modificado en los últimos años, partiendo del 2007, debido a un ascenso sostenido del flujo hacia el lago Enriquillo.

De acuerdo con Payano \& Medrano (2011) y Schubert (2012) existe una correlación geológica que permite un drenaje, aún por cuantificar, del lago Sumatre o Azuei de Haití hacia el lago Enriquillo. No parece que este drenaje sea corresponsable del aumento del nivel de las aguas del lago Enriquillo ya que de ser así esto sería una condición geológica que probablemente data desde el nacimiento de la cuenca hidrográfica. Martínez (2010) expresa que un movimiento telúrico podría tener un impacto en el lago Sumatre y verter sus aguas inundando el municipio de Jimaní, lo cual podría prácticamente unir los dos lagos (Sumatre y Enriquillo). Por su parte, Cocco-Quezada (2009) también ha expresado que en caso de desborde del lago Sumatre, este vertería sus aguas sobre el lago Enriquillo, lo cual también significa la unión de ambos lagos. La topografía del área sugiere que esto es muy poco probable.

Payano \& Medrano (2011) indican que un aumento en la sedimentación causada por la deforestación es también responsable de la crecida del lago Enriquillo. En este sentido, Romero \& Poteau (2011) indican que aunque se han producido algunos cambios en la cobertura del suelo del área entre 1986 y 2010, estos han sido mínimos. 


\section{Desvío Del YAQUe DeL SuR}

Esta hipótesis ha sido discutida en numerosos talleres celebrados en la República Dominicana. Algunos investigadores afirman que el desvío del río Yaque del Sur parece tener un impacto en el aumento del nivel del lago Enriquillo, a través del Canal Cristóbal, el cual recibió desagüe de otros canales para eventualmente drenar en el lago Enriquillo (Cocco-Quezada, 2009, Gil et al., 2012). Como resultado de esta investigación se ha podido confirmar que el canal Cristobal está siendo utilizado mayormente para riego y la mayor parte de sus aguas no llegan al lago Enriquillo.

\section{INFLUENCIA DEL CLIMA}

Varios autores han vinculado el crecimiento reciente del Lago Enriquillo al cambio climático (Valenzuela 2009). Por su parte, Herrera \& Orrego (2011) sostienen la posibilidad de que el alza en el nivel de las aguas del lago Enriquillo tenga su origen en la elevación de los océanos, los cuales han alcanzado cerca de $0.6 \mathrm{~m}$, a causa del calentamiento global y derretimiento de algunas capas de hielo. No se ha podido obtener datos en la República Dominicana que comprueben esta teoría.

Por otro lado, Gil et al. (2012) exponen una vía muy distinta para la influencia oceánica en el fenómeno del lago. Indican que de acuerdo con los hallazgos del equipo de investigadores del INTEC y City College of New York (Quintana et al., 2013), el fenómeno tiene en parte explicación con el cambio hidro-climático y el calentamiento de los océanos, lo cual está provocando mayor evaporación en el mar, aumentando la nubosidad y por ende las precipitaciones verticales y horizontales (por nubes) y toda la producción de agua dulce, la cual se acumula en la cuenca endorreica del lago Enriquillo.

Otras hipótesis ligadas al clima que han sido planteadas incluyen: La influencia de los centros mundiales de modificación de los climas como son el ENOS (El Niño, Oscilación del Sur) y la OAN 
(Oscilación del Atlántico Norte), (Cocco-Quezada, 2009); La escasa evaporación de las aguas debido a la prolongación de los inviernos (Valenzuela, 2009) y la proximidad a la astenósfera (la zona superior del manto terrestre que está inmediatamente debajo de la litósfera), provocando un aumento de la temperatura del agua causando un vapor que tendería a empujar las capas sedimentarias, elevando el nivel del agua o el nivel del fondo de los lagos (Martínez, 2010). Sin embargo, la comprobación de estas hipótesis climáticas se ven dificultadas por los datos disponibles sobre clima. Schubert (2012) expresa preocupación debido al hecho de que en el área de estudio no hay suficientes estaciones climatológicas.

\section{TeCtónica}

Algunos investigadores (p. ej. G. Rosado) han expresado que la tectónica podría tener una gran influencia en este fenómeno. Partiendo del hecho de que los estudios geológicos (específicamente de geología estructural y tectónica) han estado ausentes en las investigaciones del área y de que se necesita entender su dinámica geológica, aunque esta hipótesis necesita de estudios más profundos.

\section{Materiales y métodos}

Para este proyecto de investigación, la metodología aplicada en su desarrollo fue estructurada en tres fases. La primera fue la de recolección de datos históricos sobre la hidrología, hidrogeología y geología del área de estudio. Se utilizaron mapas geológicos a escala 1:50,000 para conocer las formaciones geológicas, el tipo de rocas y sus características. Se recopilaron datos climáticos del área a través de la Oficina Nacional de Meteorología (ONAMET). También se recopilaron datos hidrológicos e hidrogeologógicos a través del proyecto "Estudio Hidrogeológico de la zona del lago Enriquillo, determinación de las causas del aumento del nivel de sus aguas e intervenciones requeridas para su control" del INTEC. 
Durante la segunda fase se hizo un viaje de reconocimiento al área de estudio para localizar los ríos y canales en la parte oriental del lago Enriquillo. Se procedió a marcar cada uno utilizando un GPS Garmin GPSmap 62sc.

La tercera fue la fase de toma de mediciones. Esto se hizo de la siguiente manera: Se realizaron ocho viajes de campo en las diferentes estaciones del año para medir el flujo de aguas en la parte oriental del lago Enriquillo utilizando un medidor de caudal (FP111 Flow Probe, 3'- 6'). Figura 4. El flujo o caudal, es la cantidad de agua que pasa a través de una sección del rio o canal por unidad de tiempo. Se calcula multiplicando la velocidad del agua $(\mathrm{m} / \mathrm{s})$ por el área de la sección específica $\left(\mathrm{m}^{2}\right)$, y el resultado es el volumen $\left(\mathrm{m}^{3} / \mathrm{s}\right)$.

Figura N. ${ }^{\circ} 4$

Caudalímetro FP311 Global Water Flow Probe

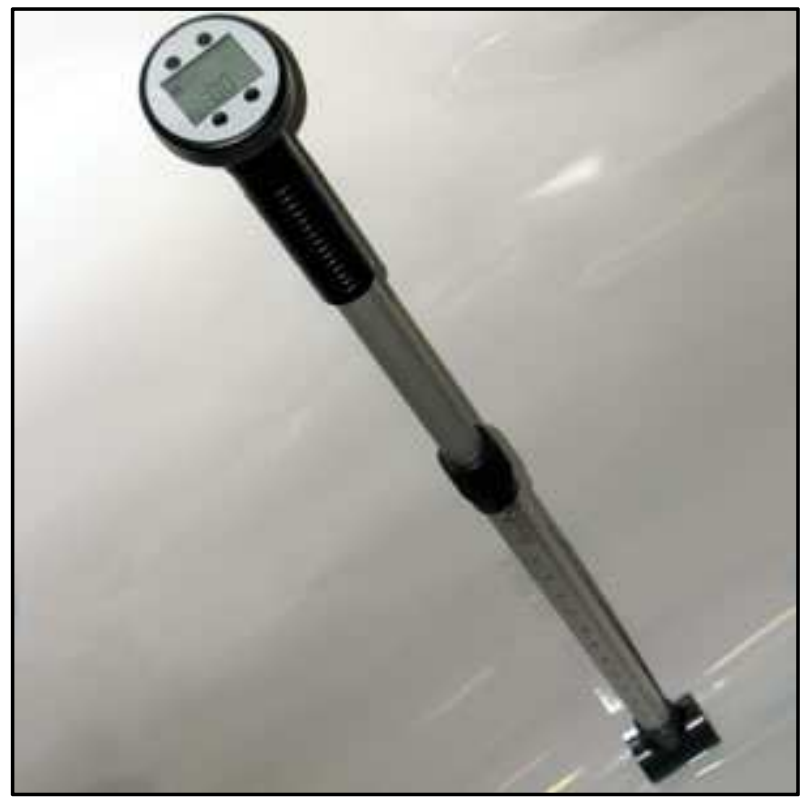

434 Ciencia y Sociedad 2015; 40(2): 425-448 
Aporte de fuentes de aguas superficiales en la parte Este del Lago Enriquillo y el impacto de esas aguas en el crecimiento del lago

Esta investigación se concentró en el aporte de las aguas superficiales del lago Enriquillo desde la parte oriental. Véase la figura N. ${ }^{\circ}$.

Figura N. ${ }^{\circ} 5$

Canales de la parte oriental del lago Enriquillo

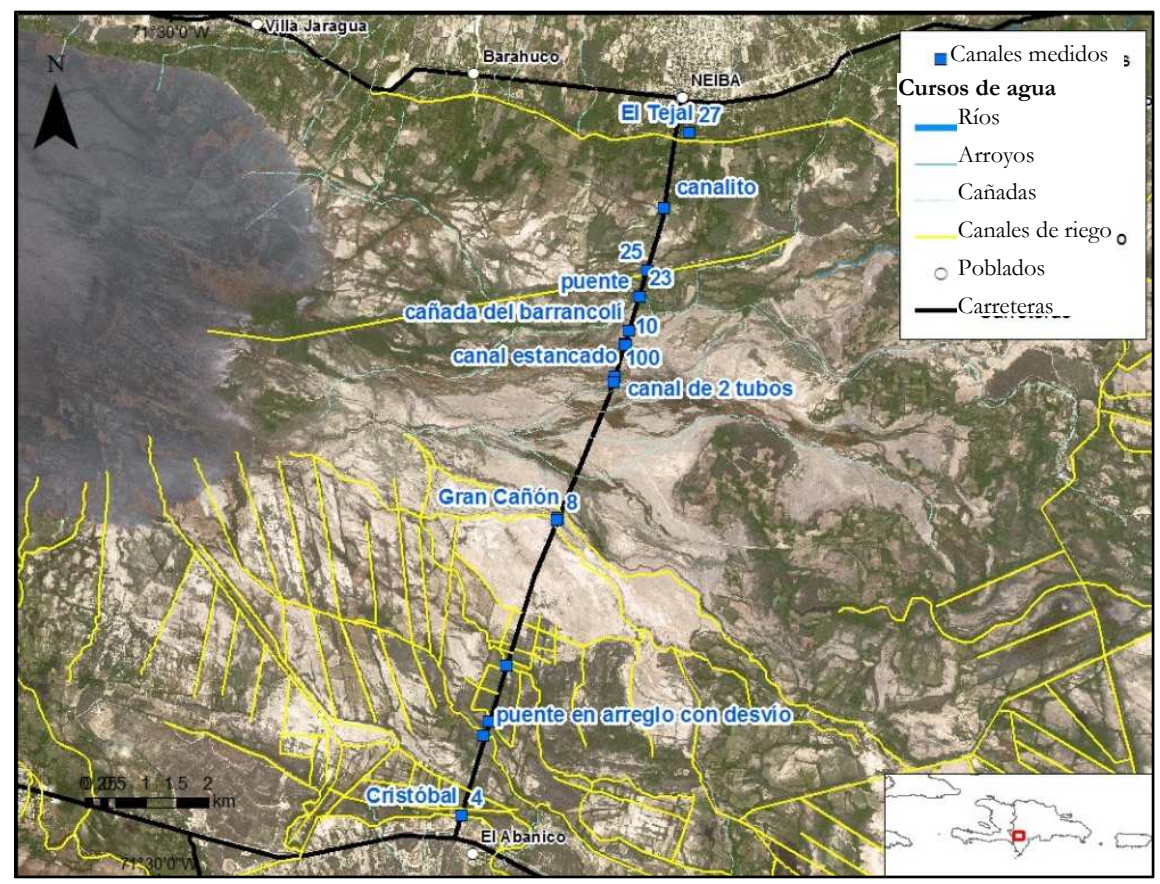

Los datos obtenidos fueron comparados con otros datos hidrológicos obtenidos a través de la Oficina Nacional de Meteorología (ONAMET) y el del equipo de investigadores del INTEC y el City College of New York. Para comparación con nuestro estudio se utilizaron como base datos obtenidos en la medición de caudales en la parte oriental del lago a través del proyecto "Estudio hidrogeológico de la zona del lago Enriquillo, determinación de las causas del aumento del nivel de sus aguas e intervenciones requeridas para su control" (FONDOCYT), el cual está en su fase final por parte del INTEC. Se utilizó el software ArcGIS para la elaboración de mapas temáticos del proyecto. 
Para la toma de mediciones de los caudales se procedió de la siguiente manera: Se marcó la localización del canal utilizando un GPS. Luego se midió el ancho del canal utilizando una cinta métrica y de inmediato se midió la profundidad del canal en el centro, en el costado izquierdo y el costado derecho del mismo y se tomó la profundidad promedio para la calculación. Luego se tomaron tres (3) medidas de velocidad del flujo de agua en cada segmento y a diferentes profundidades. (Figuras 6, 7, 8). En algunas ocasiones se tomaron cuatro o cinco mediciones debido a inconsistencias en la lectura del caudalímetro. Se calculó el promedio de la profundidad del canal, así como el promedio de las velocidades de los flujos. Para calcular el área del río se usó la siguiente ecuación:

$$
A=P_{X} L
$$

Donde $A=$ Área

$$
\begin{aligned}
& P=\text { Profundidad promedio } \\
& L=\text { Ancho }
\end{aligned}
$$

Para el "Gran Cañón" la profundidad promedio fue de 0.44 metros, el ancho 10.2 metros, y el área 4.45 metros cuadrados.

Para calcular la descarga total del río se usó la siguiente ecuación:

$$
Q=V A
$$

Donde $Q=$ Descarga total

$V=$ Velocidad promedio

$A=$ Área

Para el "Gran Cañón”

$$
\begin{gathered}
V=0.26 \mathrm{~m} / \mathrm{s} \\
A=4.45 \mathrm{~m}^{2} \\
Q=1.15 \mathrm{~m}^{3} / \mathrm{s}
\end{gathered}
$$


Aporte de fuentes de aguas superficiales en la parte Este del Lago Enriquillo y el impacto de esas aguas en el crecimiento del lago

Figura N. ${ }^{\circ} 6$

Medición del ancho de río

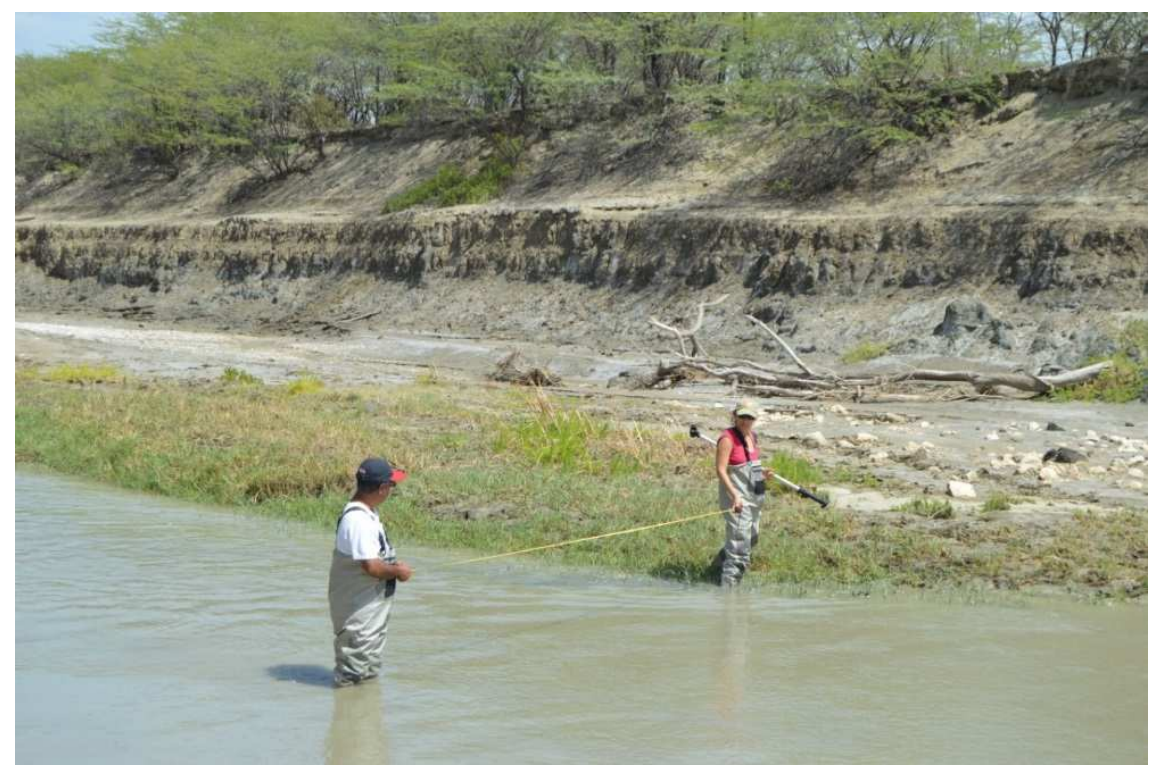

Figura N. ${ }^{\circ} 7$

Medición del ancho de canal

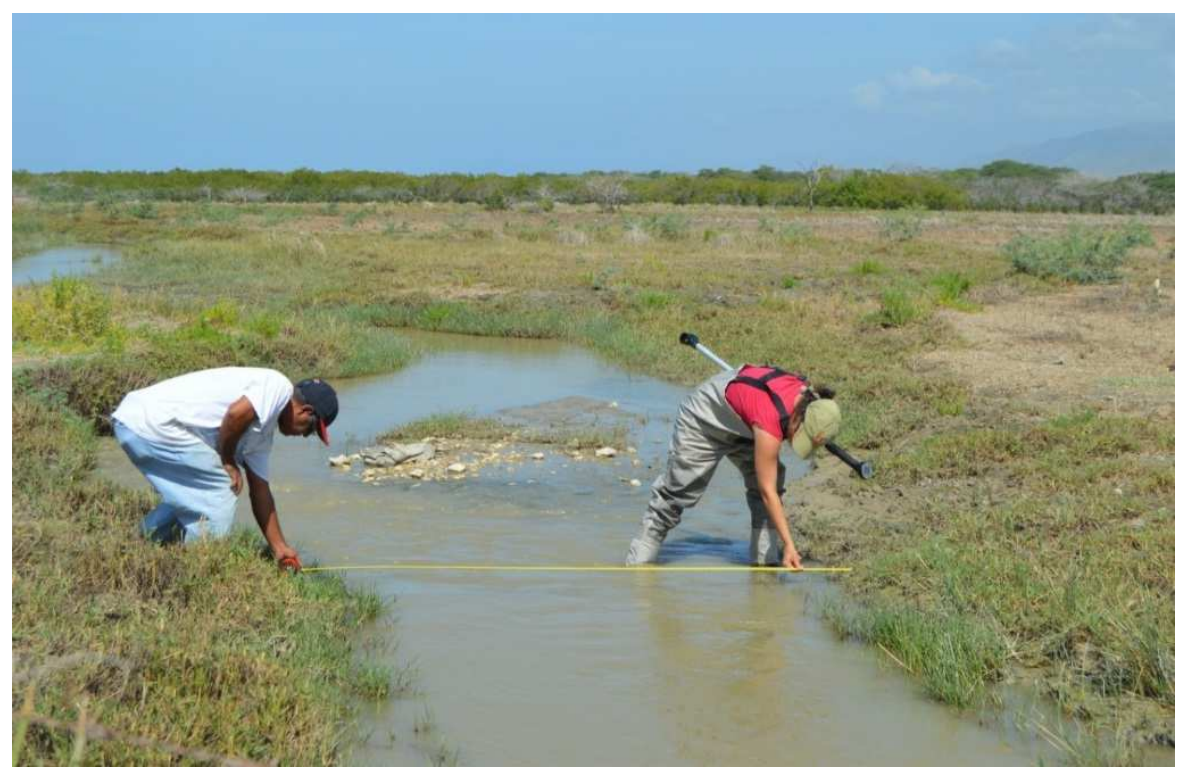

Ciencia y Sociedad 2015; 40(2): 425-448 437 
Figura N. ${ }^{\circ} 8$

Medición del flujo de canal

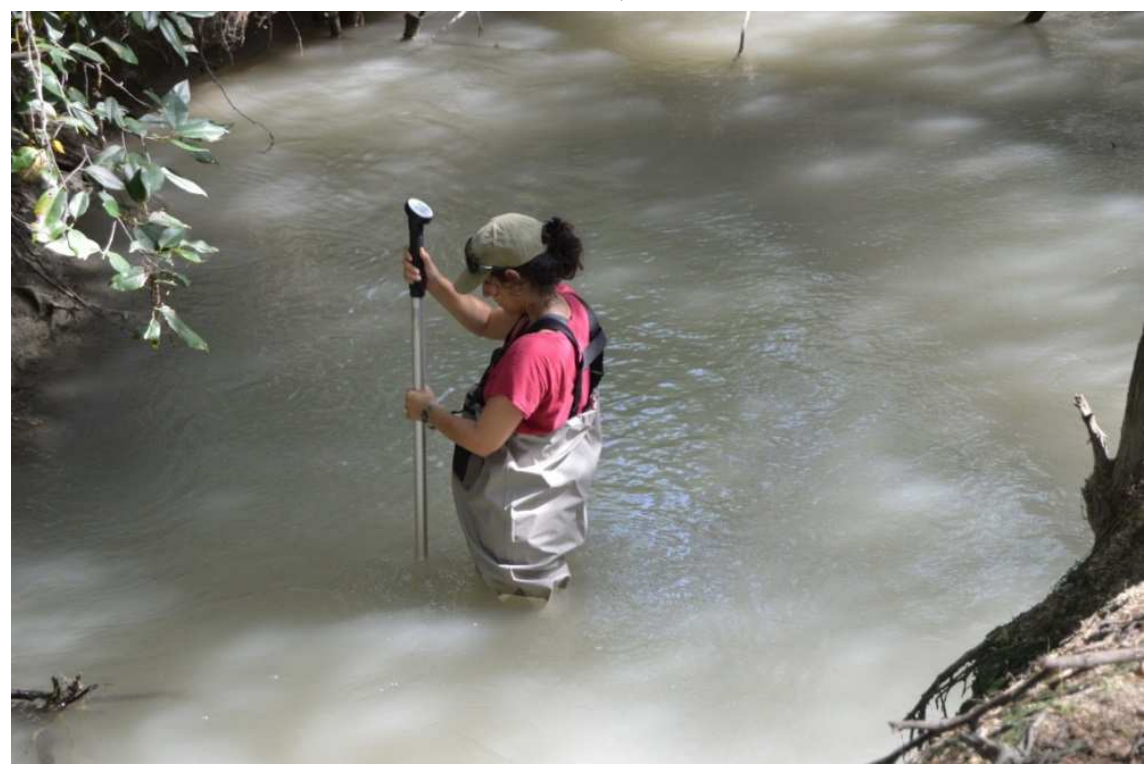

\section{Discusión}

La figura N..$^{\circ}$ y la tabla N. ${ }^{\circ} 1$ muestran claramente que para el área de estudio el año 2013 fue de extrema sequía en comparación a los datos obtenidos desde el 2000. Durante el 2013 la precipitación fue de unos $306.3 \mathrm{~mm}$, mientras que para el 2012 la precipitación fue de unos $1037.3 \mathrm{~mm}$. La Oficina Nacional de Meteorología (ONAMET) no reportó datos de precipitación para el mes de enero del 2013. Durante ese mismo período, es decir durante el mes de enero, del 2014 la precipitación fue de unos $7.5 \mathrm{~mm}$. Durante el mes de febrero del 2013 ONAMET registró una precipitación de $12.1 \mathrm{~mm}$ y durante el mismo mes, pero en el 2014 la precipitación fue de $0.9 \mathrm{~mm}$, habiendo mostrado una baja significativa para este período. No se pudo obtener datos de precipitación para el resto del 2014, pero las experiencias de los viajes de campo, además de la bajada del nivel de las aguas del lago Enriquillo, sugieren que la precipitación del 2014 fue menor a la del 2013. 
Aporte de fuentes de aguas superficiales en la parte Este del Lago Enriquillo y el impacto de esas aguas en el crecimiento del lago

Tabla N. ${ }^{\circ} 1$

Precipitación anual (mm). Estación de Jimaní

\begin{tabular}{|c|c|c|c|c|c|c|c|c|c|c|c|c|c|}
\hline AÑO & ENE & FEB & MAR & ABR & MAY & JUN & JUL & AGO & SEP & OCT & NOV & DIC & Total \\
\hline $\mathbf{2 0 0 0}$ & 15.9 & 4.1 & 1.7 & 88.2 & 165.7 & 7.5 & 11.5 & 40.1 & 250.6 & 181.9 & 6.5 & 60.2 & 833.9 \\
\hline $\mathbf{2 0 0 1}$ & 5.7 & 0.5 & 51.1 & 39.5 & 58.0 & 19.9 & 16.3 & 1.4 & 115.5 & 41.4 & 7.0 & 132.1 & 488.4 \\
\hline $\mathbf{2 0 0 2}$ & 0.6 & 3.7 & 82.8 & 147.3 & 88.3 & 87.5 & 5.8 & 16.3 & 23.3 & 10.7 & 35.6 & 8.2 & 510.1 \\
\hline $\mathbf{2 0 0 3}$ & 17.6 & 5.4 & 26.0 & 119.7 & 27.8 & 12.3 & 6.9 & 50.6 & 105.9 & 164.4 & 68.4 & 49.7 & 654.7 \\
\hline $\mathbf{2 0 0 4}$ & 13.0 & 8.9 & 79.5 & 71.4 & 312.6 & 11.2 & 77.2 & 6.9 & 131.4 & 155.7 & 2.0 & 2.1 & 871.9 \\
\hline $\mathbf{2 0 0 5}$ & 2.6 & 0.0 & 1.5 & 48.5 & 131.8 & 84.4 & 105.5 & 41.0 & 240.3 & 257.4 & 0.4 & 15.9 & 929.3 \\
\hline $\mathbf{2 0 0 6}$ & 0.0 & 53.7 & 52.7 & 167.0 & 99.4 & 200.7 & 11.7 & 152.4 & 58.9 & 101.1 & 65.9 & 0.4 & 963.9 \\
\hline $\mathbf{2 0 0 7}$ & 0.0 & 0.5 & 57.2 & 11.3 & 147.7 & 18.9 & 104.1 & 161.9 & 116.7 & 225.8 & 231.9 & 8.0 & 1084.0 \\
\hline $\mathbf{2 0 0 8}$ & 4.8 & 4.2 & 17.1 & 90.6 & 64.6 & 16.3 & 13.9 & 214.4 & 175.4 & 125.5 & 27.4 & 2.9 & 757.1 \\
\hline $\mathbf{2 0 0 9}$ & 28.3 & 41.6 & 13.4 & 59.8 & 81.6 & 82.7 & 18.2 & 109.8 & 91.5 & 82.7 & 48.5 & 36.8 & 694.9 \\
\hline $\mathbf{2 0 1 0}$ & 43.0 & 3.3 & 17.6 & 42.5 & 104.5 & 89.0 & 152.4 & 65.1 & 83.5 & 114.7 & 64.0 & 27.5 & 807.1 \\
\hline $\mathbf{2 0 1 1}$ & 0.0 & 32.3 & 25.7 & 87.6 & 345.8 & 76.0 & 53.9 & 46.7 & 38.5 & 21.9 & 57.3 & 93.8 & 879.5 \\
\hline $\mathbf{2 0 1 2}$ & 12.6 & 36.6 & 158.8 & 185.4 & 92.9 & 24.7 & 72.2 & 115.6 & 33.4 & 241.3 & 59.7 & 4.1 & 1037.3 \\
\hline $\mathbf{2 0 1 3}$ & 0.0 & 12.1 & 20.0 & 25.8 & 76.8 & 17.1 & 34.3 & 14.0 & 44.5 & 47.3 & 12.7 & 1.7 & 306.3 \\
\hline $\mathbf{2 0 1 4}$ & 7.5 & 0.9 & - & - & - & - & - & - & - & - & - & - & \\
\hline PROM & 10.1 & 13.9 & 43.2 & 84.6 & 128.4 & 53.4 & 48.9 & 74.0 & 107.8 & 126.6 & 49.1 & 31.7 & \\
\hline
\end{tabular}

Fuente: Oficina Nacional de Meteorología.

Figura N. ${ }^{\circ} 9$

Precipitación anual (mm)

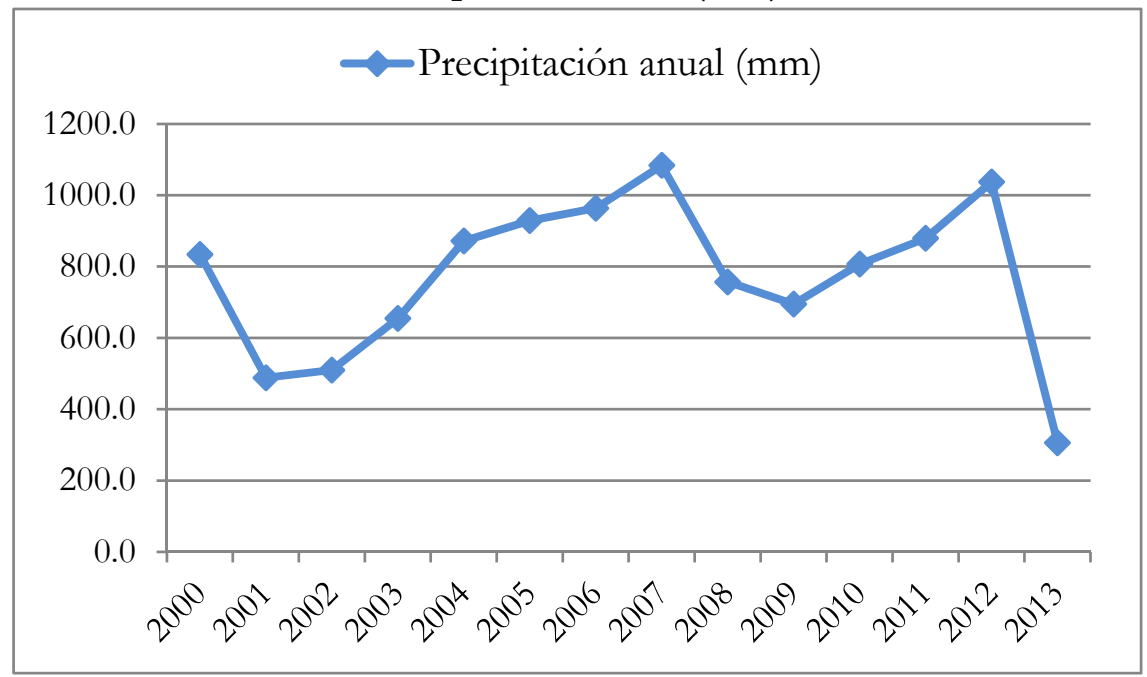

Fuente: Onamet. 


\section{Resultados}

En total se contabilizaron 8 canales en la parte oriental del lago Enriquillo. Se pudo constatar que siete de los mismos son utilizados para riego y sus aguas no drenan hacia el lago. El canal nombrado "El Gran Cañón" fue el único que no se pudo establecer si realmente drena sus aguas hacia el lago. Se calculó para este un ancho aproximado de 10.2 metros, una profundidad aproximada de $0.44 \mathrm{~m}$, y un promedio de flujo de agua de unos 1.15 metros cúbicos por segundo. Algunos canales reflejaron un flujo mínimo a principios del año, pero luego se secaron.

Se debe señalar que mientras algunos canales se secaron durante el 2014, otros nuevos canales fueron construidos para riego, obstaculizando de esa manera las calculaciones hechas sobre los canales originales en el sitio de estudio. Aun así no se sabe la fecha exacta cuando esos canales fueron hechos, siendo imposible estimar el flujo de los mismos para el año 2014.

\section{Conclusión}

Durante el año 2014 la región suroeste de la República Dominicana sufrió una sequía fuera de lo normal. Por este motivo las mediciones hechas durante este año no son representativas del sitio de estudio. También fueron hechos varios canales de riego que deberían tomarse en cuenta en estudios posteriores.

A excepción del canal nombrado como "El Gran Cañón" ninguno lleva aguas superficiales al lago. El aporte de estos al mismo sería a través de aguas subterráneas y de la evapo-transpiración. Por esto se sugiere una continuidad en la toma de mediciones e interpretaciones durante los próximos dos años a fin de obtener datos representativos sobre el aporte de las aguas superficiales desde la parte oriental del lago Enriquillo. 
Aporte de fuentes de aguas superficiales en la parte Este del Lago Enriquillo y el impacto de esas aguas en el crecimiento del lago

\section{Bibliografía}

Ali, S. T.; Freed, A. M.; Calais, E.; Manaker, D. M. \& McCann, W. R. (2008). Coulomb Stress evolution in northeastern Caribbean over the past 250 years due to coseismic, postseismic, and interseismic deformation. Geophysical Journal International, 174, 904-918.

Aquater, S. P. A. (2000). Estudio bidrogeológico nacional: estudio bidrogeológico del Valle de Neiba. (Memoria $N .^{\circ}$ 5). Recuperado de http://mapas.igme.es/sgn/hidro/memorias/Estudio $\% 20$ Hidrogeol $\%$ C3\%B3gico $\% 20$ del $\% 20$ Valle $\% 20 \mathrm{de}^{2} \% 20$ Neiba .pdf

Bakun, W. H., Flores, C., Ten, B. \& Uri, S. (2002). Significant Earthquakes on the Enriquillo Fault System, Hispaniola. 1500-2010: Implications for Seismic Hazard. Bulletin of the Seismological Society of America, 102(1), 18-30. doi: 10.1785/ 0120110077.

Bilharm, R. (2010). Structural geology. Invisible faults under shaky ground. Nature Geosciences. Recuperado de http://www.nature.com/ ngeo/journal/v8/n3/index.html

Buck, D. G.; Brenner, M.; Hodell, D. A.; Curtis, J. H.; Martin, J. B.; \& Pagani, M. (2005). Physical and chemical properties of hypersaline Enriquillo Lake, Dominican Republic. Recuperado de http://people.earth.yale.edu/sites/default/files/files/Pagani/ 4_2005\%20Buck\%20et\%20al.pdf

Calais, E., Freed, A. F., Mattioli, G., Amelung, F., Jónsson, S., Jansma, P., Hong, S-H., Dixon, T., Prépetit, C. \& Momplaisir, R. (2010). Transpressional rupture of an unmapped fault during the 2010 Haiti earthquake. Nature Geoscience, 3, 794-799.

Calais, E., Mazabraud, Y., Mercier de Lénay, B., Mann, P. Mattioli, G. \& Jansma, P. (2002). Strain partitioning and fault slip rates in the northeastern Caribbean from GPS measurements. Geophys, Res. Lett., 29(18), 18-56, doi: 10.1029/2002GL015397. 
Coco Quezada, A. (2009). El ciclo bidrológico del lago Enriquillo y la crecida extrema del 2009. Recuperado de http://www.acq weather.com/EL\%20CICLO $\% 20$ HIDROLOGICO $\% 20$ DEL\%20LAGO\%20ENRIQUILLO.pdf

Costilla, M. \& Udias, A. (1999). Geodinámica del límite CaribeNorteamérica. Revista Sociedad Geológica España, 12(2), 175-186.

Cowgill, E., Bernadin, T. S., Oskin, M. E., Bowles, C., Burak, M., Oliver Kreylos, K. et al. (2012). Interactive terrain visualization enables virtual fileld work during rapid scientific response to the 2010 Haiti earthquake. Geosphere, 8, 3, 1-18.

Dixon, T. H., Farina, F., DeMets, C., Jansma, P., Mann, P. \& Calais, E. (1998). Relative motion between the Caribbean and North American plates and related boundary zone deformation from a decade of GPS observations. Journal of Gepphysical Research, 103(15), 157-182.

Dolan, J. F. \& Mann, P. (Ed.). (1998). Active Strike-Slip and Collisional Tectonics of the Northern Caribbean Plate Boundary Zone. Colorado: Geological Society of America.

Dolan, J., Mann, P., De Zoeten, R., Heubeck, Ch., Shirona, J. \& Monechi, S. (1991). Sedimentologic, stratigraphic and tectonic synthesis of Eocene-Miocene Sedimentary Basins, Hispaniola and Puerto Rico. [Paper 262]. Colorado: Geological society of America Special.

Ducoudray, F. S. (2006). Y una escalera grande para bajar al lago. En A. Incháustegui, B. Delgado-Malagón (Eds.). La naturaleza dominicana: artículos publicados en el suplemento sabatino del periódico El Caribe, 1978-1989. Santo Domingo: Grupo León Jimenes.

Etypsa Group (2004). Estudio bidrogeológico nacional de la República Dominicana Fase II. Volumen 10: Resumen, conclusiones y recomendaciones. [Memoria general]. Recuperado de http://mapas. igme.es/sgn/hidro/memorias/Memoria\%20General.pdf 
Hayes, G. P.; Briggs, R. W.; Sladen, A.; Fielding, E. J.; Prentice C.; Hudnut, K.; Mann, P.; Taylor, F. W.; Crone, A. J.; Gold, R., Ito, T. \& Simons, M. (2010). Complex rupture during the 12 January 2010 Haiti earthquake. Nature Geoscience, 3, 800-805. doi: 10.1038/NGEO977.

Herrera, M., A. \& Orrego Campo, J. C. (2011). Revisión del estado de la situación de riesgo climático y su gestión en República Dominicana. [IISD Report]. Santo Domingo: Instituto Internacional para el Desarrollo Sostenible y Naciones Unidas.

Incháustegui, S. J., Gutiérrez, W., Rivas, V., Álvarez, V., Núñez de Ricart, N., Bonnelly de Calventi, I. (1977). Notas sobre la ecología del Lago Enriquillo de República Dominicana. En I. Bonnelly de Calventi (Ed.). Conservación y ecodesarrollo. Santo Domingo: Universidad Autónoma de Santo Domingo.

Jiménez Cisneros, B. \& Galizia Tundisi, J. (2012). Diagnóstico del agua en las Américas. México: Red Interamericana de Academias de Ciencias y Foro Consultivo Científico y Tecnológico.

Koehler, R. D., Mann, P. \& Brown, L. A. (2009). Tectonic geomorphology and paleoseismology of strike-slip faults in Jamaica: Implications for distribution of strain and seismic hazard along the southern edge of the Gonave microplate. Nature Geoscience, 3, 789-793 (2009). Recuperado de http://www. nature.com/ngeo/journal/v3/n11/abs/ngeo991.html.

Noble, G. K. (1929). Trailing the rhinoceros iguana. Natural History, 23(6), 541-558.

Mann, P., McLaughlin, P. P. \& Cooper, C. (1991). Geology of the Azua and Enriquillo Basins, Dominican Republic: Structure and Tectonics. Geological Society of America Special [Paper 262]. Colorado: Boulder. 
Mann, P., Taylor, F., Edwards, R. \& Ku, T. (1995). Actively Evolving Microplate Formation by Oblique Collision and Sideways Motion Along Strike-Slip Faults: An example from the Northeastern Caribbean Plate Margin. Tectonophysics, 246, 1-69.

Mann, P. et al. (2002). Oblique Collision in the Northeastern Caribbean from GPS Measurements and Geological Observations. Tectonics, 21:1057. doi: 101029/2001TC001304

Mann, P., Calais, E., Demets, Ch., Prentice, C. S. \& WigginsGrandison, M. (2008). Enriquillo-Plantain Garden Strike-Slip Fault Zone: A Major Seismic Hazard Affecting Dominican Republic, Haiti and Jamaica. (18th Caribbean Geological Conference), Santo Domingo.

Martínez, H. (2010). Consideraciones acerca del riesgo de inundación del poblado de Jimaní. Riesgo de Inundación debido al rebose del lago Saumätre o Azuei hacia el Lago Enriquillo. [Documento inédito].

Medrano, R. \& Medrano, O. (2011). Análisis de la situación actual del lago Enriquillo de la República Dominicana. Recuperado de www.agua.imdea.org

Prentice, C. S., Mann, P., Crone, A. J., Gold, R. D., Hudnut, K. W., Briggs, R. W., Koehler, R. D. \& Jean, P. (2010). Seismic Hazard of the Enriquillo-Plantain Garden fault in Haiti inferred from Palaeoseismology. Nature Geoscience, 3, 789-793.

Quintana, C., León, Y., Miniño, V., González, J., Comarazamy, D. (2013). Estudio bidrogeológico de la zona del Lago Enriquillo, determinación de las causas del aumento de nivel de sus aguas e intervenciones requeridas para su control. (Proyecto FONDOCYT). Instituto Tecnológico de Santo Domingo \& City College of New York. [Documento inédito] 
Aporte de fuentes de aguas superficiales en la parte Este del Lago Enriquillo y el impacto de esas aguas en el crecimiento del lago

Risk Management Solutions, Inc. (2010). RMS FAQ: 2010 Haiti Earthquake and Caribbean Earthquake Risk. Recuperado de http://www.mona.uwi.edu/cardin/virtual_library/docs/1342 /1342.pdf

Romero Luna, E. J. \& Poteau, A. D. (2011). Water Level Fluctuations of Lake Enriquillo and Lake Saumatre in Response to Environmental Changes. (Tesis de maestría). New York, Cornell University.

Sociedad Dominicana de Geología \& Sociedad Dominicana de Sismología e Ingeniera Sísmica. (2010). Simposio Terremoto de Haiti: Una alerta para la Hispaniola. Hotel Santo Domingo, Santo Domingo.

Yule, D. \& Sieh, K. (2003). Complesity of the San Andreas Falut Near San Gorgonio Pass: Implications of Large Earthquakes. Journal of geophysical Research, 108, N. ${ }^{\circ}$ B11, 2548, doi: 10. 1029/2001JB000451. 


\section{Virgilio Antonio Miniño Mejía, Ph. D.}

Doctorado en Ciencias Ambientales del Centro de Graduados de la Universidad de la Ciudad de Nueva York, con especialidad en Hidrogeología. También posee una maestría en Geología Estructural del City College of New York, desde donde también obtuvo su título de geólogo. Fue profesor universitario por cuatro años en la universidad City College of New York y por dos años en el Bergen College of New Jersey. El Dr. Miniño hizo trabajo de investigación geológica en el área de Catskill del estado de Nueva York durante los años 1992-1997 para el City College of New York. En el presente es el investigador principal del proyecto de investigación "Determinación de las Influencias Neotectónicas, Fallas, Fracturas y otras estructuras geológicas, sobre el continuo crecimiento del nivel de las aguas del Lago Enriquillo" financiado por FONDOCYT.

Correo electrónico: virgilio.minino@intec.edu.do 
Aporte de fuentes de aguas superficiales en la parte Este del Lago Enriquillo y el impacto de esas aguas en el crecimiento del lago

\section{Yolanda León, Ph. D.}

Profesora-investigadora del INTEC, República Dominicana. Licenciada en Biología de la Universidad Autónoma de Santo Domingo, República Dominicana (1997), máster en ciencias de la Universidad de Florida, Estados Unidos (2000) y doctora en Ciencias de la Universidad de Rhode Island, Estados Unidos (2004). Posee 14 años de experiencia como profesora universitaria e investigadora. Actualmente se desempeña como profesora investigadora y como encargada del Laboratorio de Percepción Remota del INTEC. Es autora de 8 artículos científicos y ha presentado varias comunicaciones a eventos científicos.

Correo electrónico: ymleon@intec.edu.do

\section{Aida Rosario}

Posee el grado de ingeniera civil del Instituto Tecnológico de Santo Domingo (INTEC). Actualmente cursa en el programa de maestría en medio ambiente del INTEC. Actualmente se desempeña como asistente técnico del despacho del Ministro de Medio Ambiente y Recursos Naturales de República Dominicana.

Correo electrónico: aida.rosario@gmail.com 


\section{Cándido Quintana, Ph. D.}

Es ingeniero Termoenergético, Dr. en Ciencias Técnicas y profesor titular de la Universidad Central de las Villas, Cuba. Tiene estudios de postgrado por el Instituto Politécnico de San Petersburgo, Rusia. Ha cursado estudios posdoctorales en la Universidad Politécnica de Cataluña, España, y en la Universidad de Ghent, Bélgica. Ha sido consultor del Imperial College of Lodon University para proyectos de energía y medio ambiente. Ha laborado como representante por Cuba en varios proyectos internacionales. Actualmente labora como profesor-investigador del Área de Ciencias Básicas y Ambientales del INTEC. Fue líder científico del proyecto "Potencial de inserción del INTEC en el Mecanismo de Desarrollo Limpio del Protocolo de Kioto".

Correo electrónico: candido.quintana@intec.edu.do

Recibido: 28/03/2015 Aprobado: 25/05/2015 